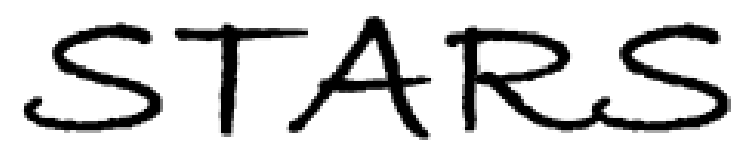

University of Central Florida

STARS

Faculty Bibliography 2000s

Faculty Bibliography

$1-1-2009$

\title{
Effect of surface tension on nanotube nanofluids
}

\author{
Ranganathan Kumar \\ University of Central Florida \\ Denitsa Milanova \\ University of Central Florida
}

Find similar works at: https://stars.library.ucf.edu/facultybib2000

University of Central Florida Libraries http://library.ucf.edu

This Article is brought to you for free and open access by the Faculty Bibliography at STARS. It has been accepted for inclusion in Faculty Bibliography 2000s by an authorized administrator of STARS. For more information, please contactSTARS@ucf.edu.

\section{Recommended Citation}

Kumar, Ranganathan and Milanova, Denitsa, "Effect of surface tension on nanotube nanofluids" (2009).

Faculty Bibliography 2000s. 1759.

https://stars.library.ucf.edu/facultybib2000/1759

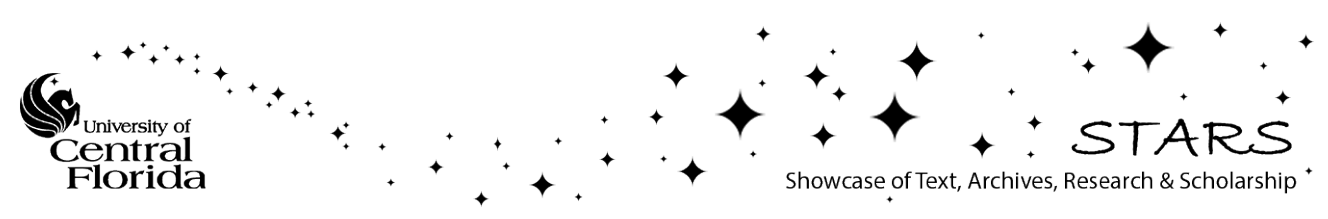




\section{Effect of surface tension on nanotube nanofluids}

Cite as: Appl. Phys. Lett. 94, 073107 (2009); https://doi.org/10.1063/1.3085766

Submitted: 13 November 2008 . Accepted: 06 January 2009. Published Online: 18 February 2009

Ranganathan Kumar, and Denitsa Milanova

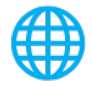

View Online

\section{ARTICLES YOU MAY BE INTERESTED IN}

Effects of nanoparticle deposition on surface wettability influencing boiling heat transfer in nanofluids

Applied Physics Letters 89, 153107 (2006); https://doi.org/10.1063/1.2360892

Effect of nanoparticles on critical heat flux of water in pool boiling heat transfer Applied Physics Letters 83, 3374 (2003); https://doi.org/10.1063/1.1619206

Surface Tension and Viscosity of Aluminum Oxide Nanofluids

AIP Conference Proceedings 1207, 460 (2010); https://doi.org/10.1063/1.3366409

\section{Applied Physics Letters}

Mid-IR and THz frequency combs special collection

Read Now! 


\title{
Effect of surface tension on nanotube nanofluids
}

\author{
Ranganathan Kumar ${ }^{\mathrm{a})}$ and Denitsa Milanova \\ Department of Mechanical, Materials and Aerospace Engineering, University of Central Florida, \\ Orlando, Florida 32816, USA
}

(Received 13 November 2008; accepted 6 January 2009; published online 18 February 2009)

\begin{abstract}
This letter presents heat transfer results that single-walled carbon nanotube (CNT) suspensions in a boiling environment can extend the saturated boiling regime and postpone catastrophic failure of the material even further than previously reported if the surface tension of the nanofluid is carefully controlled. The maximum enhancement in the critical heat flux is nearly four times for a surfactant to CNT concentration ratio of 1:5. The experimental results show that the material burnout is a strong function of the relaxation of the nanofluid surface tension with the base fluid. (C) 2009 American Institute of Physics. [DOI: 10.1063/1.3085766]
\end{abstract}

Suspensions of less than $50 \mathrm{~nm}$ metallic and oxide particles and carbon nanotubes (CNTs) in base fluids, called smart or nanofluids, have stimulated immense interest in potential thermal transport applications. Although there is much controversy in the enhancement in thermal conductivity in single phase flows, ${ }^{1}$ the significant increase in the critical heat flux (CHF) enhancement in pool boiling is undisputed. ${ }^{2}$

The conductivity studies performed in alumina nanofluids prepared differently in three different laboratories ${ }^{2-4}$ showed incremental change to about $30 \%$ improvement. The discrepancies between different groups could be attributed to particle size distribution, peculiarities of nanoparticle synthesis, nature of stabilizing agents, surface charge, $p \mathrm{H}$, and differences in the adopted measurement techniques. Our recent analytical work ${ }^{5}$ to resolve the controversy showed that one of the previously proposed mechanism, Brownian motion, can increase the conductivity of the nanofluid by $6 \%$ primarily through random walk motion and not only through diffusion. Recently, a theoretical model was presented based on the chain formation of CNTs in the base fluid to show that the thermal conductivity can be significantly enhanced in CNT nanofluids. ${ }^{6}$

Our group has consistently measured three-digit increases in the burnout heat flux (BHF) in pool boiling studies $^{7-9}$ of silica nanofluids. In an isolated experiment, we delineated the effects of nanofluid in suspension without the effect of deposition on the heating surface and showed ${ }^{9}$ that the BHF was enhanced by 35\%-50\% regardless of the particle diameter and $p \mathrm{H}$ of the solution. Hence we concluded that in the experiments with three-digit increases in BHF, the deposition on the heating wire played a major role. The formation of wire coating contributed to more nucleation sites for bubble initiation. You et al. ${ }^{10}$ performed $\mathrm{Al}_{2} \mathrm{O}_{3}$ nanofluid studies on a flat plate to show a threefold increase in CHF. Das et al. ${ }^{11}$ reported the deterioration of boiling heat transfer in the presence of nanoparticles.

In the literature, an enhancement of CNTs has been reported in boiling with significant deviation in the results. This letter will show that such a range in enhancement is possible and would depend on the concentration of the surfactants needed to suspend the CNTs. Specifically, it will be shown that for different nanotube concentrations, the burnout

\footnotetext{
${ }^{a)}$ Electronic mail: rnkumar@mail.ucf.edu.
}

of the material could be postponed if relaxation of the surface tension of the nanofluid, i.e., the difference in the surface tension between the nanofluid and surfactant solution, is carefully controlled.

Single-walled CNTs (SWNTs) used in the nanofluids are synthesized by a high pressure carbon monoxide reactor, which is a process that produces $100 \%$ high-purity SWNTs with an ash content of less than 5 wt \%. SWNTs were acquired in powder form and are a product of CNI Nanotechnologies (Dallas, Texas). Both samples are purified and large catalyst particles are removed a priori for the nanofluid preparation described below. Treatment of bundles of CNTs only with reactive reagents could often result in functionalization of outermost nanotubes, while tubes within the bundle remain intact. It has been reported that the acid treatment of multiwalled CNTs enhances their stability in water by converting hydrophobic walls to hydrophilic. ${ }^{12}$ Here we propose two methodologies for the preparation of SWNTsnanofluid. Initially, nanotubes have been functionalized with high concentration hydrochloric acid $(\mathrm{HCl})$, sonicated, and coated with surfactant (i.e., covalent+ noncovalent). The second protocol is a single-step solubilization in a surfactant solution (only noncovalent). Both of these methods gave satisfactory results in terms of creating a homogeneous dispersion. Differences arise with respect to heat transfer characteristics, which are discussed below.

Pristine SWNT bundles were refluxed with 36 wt \% concentration $\mathrm{HCl}$ at $100{ }^{\circ} \mathrm{C}$ for $12 \mathrm{~h}$. The ratio of nanotubes and solvent was $1: 50$ by weight. Hydrochloric acid was chosen as a reactive reagent because it removes catalytic particles without reducing the length of the tubes or damaging the sidewalls. In addition, $\mathrm{HCl}$ facilitates covalent functionalization of SWNTs with $\mathrm{H}^{+}$. Other acids $\left(\mathrm{HNO}_{3}\right.$ and $\mathrm{HNO}_{3}: \mathrm{H}_{2} \mathrm{SO}_{4}$ ), tried as refluxing mixtures, were too harsh and damaged the structure. CNTs were separated from the acid by centrifugation for $1 \mathrm{~h}$ and left to dry. The surfactant solution was prepared by sonicating $\mathrm{NaDBS} /$ water mixture in a cup-and-horn-type ultrasonicator for $1 \mathrm{~h}$. Different surfactant: CNT weight ratios were used and the results are reported. All surfactant concentrations did not exceed the critical micelle concentration (CMC).

Figure 1(a) shows a transmission electron microscope (TEM) image of a sample of pristine nanotubes ( $p$-SWNTs) at low magnification. Untreated SWNTs have a great ten- 


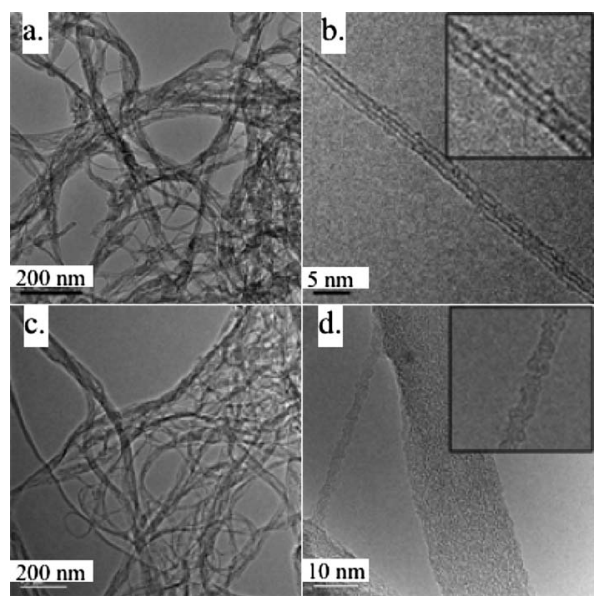

FIG. 1. TEM images of (a) pristine SWNTs (scale bar of $200 \mathrm{~nm}$ ), (b) magnified image of bundled SWNTs (scale bar of $5 \mathrm{~nm}$ ), (c) NaDBS-coated SWNTs (scale bar of $200 \mathrm{~nm}$ ), and (d) magnified image of NaDBS-coated SWNTs (scale bar of $10 \mathrm{~nm}$ ).

dency to align and bundle in ropes that can be observed in Fig. 1(b). p-SWNTs have smooth walls as opposed to surfactant adhered nanotubes [Figs. 1(c) and 1(d)]. The association of NaDBS is accompanied by the creation of wall roughness. Individual SWNT covered with surfactant molecules is shown on Fig. 1(d). The micrograph clearly shows roughness on the sidewalls, which prompts good adsorption. The type and degree of CNTs covalent and noncovalent surface treatment cause changes in the electronic structure of the tubes. Therefore, it is important to determine the appropriate chemical manipulation and the best surfactant to SWNTs proportion with respect to boiling heat transfer performance. A discussion of the pool boiling results follows.

The pool boiling experiment was carried out according to a procedure provided by the authors. ${ }^{8}$ One significant modification is related to resistance measurements across the $\mathrm{NiCr}$ wire. A four point method for measuring the resistance has been adopted since this technique eliminates problems related to determining lead and contact resistances. To serve the purpose, a second set of lead wires (sense wires) has been attached to the NiChrome wire and the potential drop is determined across a smaller length $(\sim 40 \mathrm{~mm})$ than that determined by main lead copper wires. The sense lines are connected to the multimeter and draw minimal current, resulting in accurate voltage measurements. The current measurements were taken at $0.1 \mathrm{~A}$ increments and $1 \mathrm{~s}$ was allowed for the time response of the wire before the potential drop was recorded. The heat flux from the wire to the saturated liquid is determined from the current, voltage, wire diameter, and length.

The resistance of the NiChrome wire is related to the temperature as per the manufacturer's (Omega) data sheet. Precise calibration for the temperatures between 100 and $150{ }^{\circ} \mathrm{C}$ was experimentally determined by immersing the wire in a thermostat bath of electrically nonconducting silicone oil. Two main parameters were varied: one is the surface tension and the other is the chemical treatment of nanotubes sidewalls (i.e., oxidized and untreated sidewalls). The concentration of SWNTs in the nanofluid is kept constant for all cases at $0.1 \%$ by volume. The two heat fluxes that are of importance are the CHF where the sudden jump in temperature occurs and the BHF at which point the wire fails.

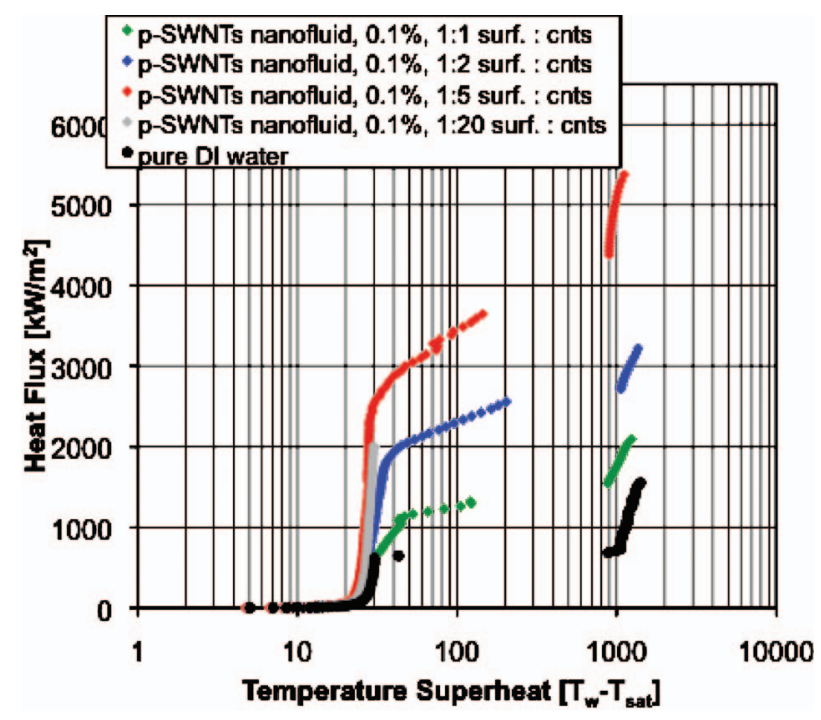

FIG. 2. (Color) Pool boiling curve for pristine SWNTs-nanofluid with various surfactant concentrations.

The results of pool boiling heat flux with superheat, which is the wire temperature minus saturation temperature of the liquid, i.e., $\left(T_{w}-T_{s}\right)$, are presented in Fig. 2. The surfactant to CNT concentration varies from 1:20 to $1: 1$ corresponding to $0.05 \times 10^{-3}-1.3 \times 10^{-3} \mathrm{~g} / \mathrm{ml}$. The CHF value for pure de-ionized (DI) water (black symbol) is $750 \mathrm{~kW} / \mathrm{m}^{2}$ where a sudden rise in temperature occurs. At the point where the wire breaks, BHF is $1500 \mathrm{~kW} / \mathrm{m}^{2}$. The results were verified by repeating the experiment multiple times; however, only one data trend is shown for clarity. The gap in the data represents the sudden jump in the temperature due to the jump in the resistance in the material calibration curve. Any data in this gap were removed due to the high uncertainty in measurements. This natural discontinuity in temperature truly represents the CHF. As the surfactant concentration ratio is increased from 1:20 to 1:5, CHF increases; however, if the concentration ratio is further increased to 1:1, the CHF drops drastically. This behavior suggests that there is a critical concentration at which CHF is maximum. The burnout behavior is similar as BHF increases to $5470 \mathrm{~kW} / \mathrm{m}^{2}$ (265\% enhancement over DI water), but again for $1: 1$ concentration ratio, it falls to $2095 \mathrm{~kW} / \mathrm{m}^{2}$. All the aforementioned heat transfer results are provided for a single-step solubilization of $p$-SWNTs in a NaBDS solution.

Before analyzing the results for a 1:5 concentration ratio, results were obtained for oxidized SWNTs $(o$-SWNT) for comparison. This case is prepared by the two-step solubilization method when the SWNTs are oxidized before dispersed in a solvent (surfactant solution). Although CHF is almost the same for both cases (not shown in figure), it is important to note that the oxidation of the CNT sidewalls affects the BHF by decreasing it from 5470 to nearly $4000 \mathrm{~kW} / \mathrm{m}^{2}$. By introducing a charge on the nanotubes, we were able to manipulate not only the dispersion but also the orientation and accumulation of SWNTs on the heated $\mathrm{NiCr}$ wire. It has been claimed ${ }^{13}$ that the deposition of nanoparticles on the heated element is the sole reason for any enhancement in CHF. In fact the deposition plays an important role in determining the $\mathrm{CHF}$ regime and the possible formation of vapor jets and columns. With an increase in the surfactant, i.e., decrease in surface tension, the deposition on the wire increases as well. Scanning electron microscopy (SEM) 


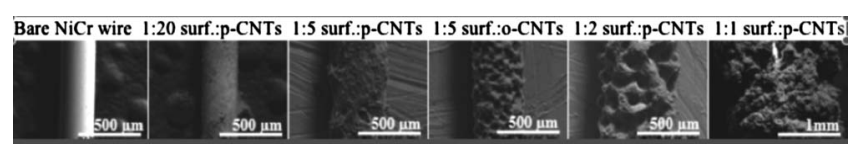

FIG. 3. SEM images of $\mathrm{NiCr}$ wires at various surfactant concentrations after pool boiling.

micrographs for all the cases discussed so far are provided in Fig. 3. However, the 1:5 concentration ratio is optimum, and maximum $\mathrm{CHF}$ and BHF do not occur when the deposition is the highest. Hence, we conclude that the heat transfer behavior is dictated not only by the deposition of nanotubes on the heated wire but also by the surface tension, which is a precursor to deposition and postponement of CHF.

In order to understand the interesting result of maximum enhancement in critical and BHF at a concentration ratio of $1: 5$, the surface tension of the DI water and the nanofluid, both in the presence of NaBDS surfactant, is plotted against concentration in Fig. 4. The surface tension measurements were made using SITA tensiometer using the bubble pressure method. With no nanotubes suspended in the solution, the surface tension of DI water with only the surfactant steadily decreases until CMC is reached. This is in agreement with the work of Wasekar and Manglik ${ }^{14}$ who mixed the surfactant, sodium dodecyl sulfate (SDS), in water to evaluate the nucleate pool boiling performance. They found that the boiling performance was enhanced by the presence of SDS with an early onset of nucleate boiling. They found an optimum level of enhancement at or near CMC of the surfactant. The enhancement decreased in high concentration solutions.

When the CNTs are added to DI water with surfactant, for the first three low concentration ratios of 1:20, 1:10, and $1: 5$, the surface tension profile of the solution is flat and is close to that of DI water. This is due to the adsorption of ionic surfactant $(\mathrm{NaDBS})$ on the SWNTs that results in the stabilization of the nanotubes in aqueous solution. The surfactant hydrophilic head orients onto the water phase, while the hydrophobic tail is bound to the nanotube walls. This leaves the surface tension of the nanofluid at the three lowest concentrations the same as that of DI water. At concentration ratios of $1: 4$ through $1: 1$, the surface tension steadily decreases as more of the surfactant molecules mix with DI water. Due to the adsorption characteristics of the surfactant on the nanotubes, the difference between the nanofluid sur-

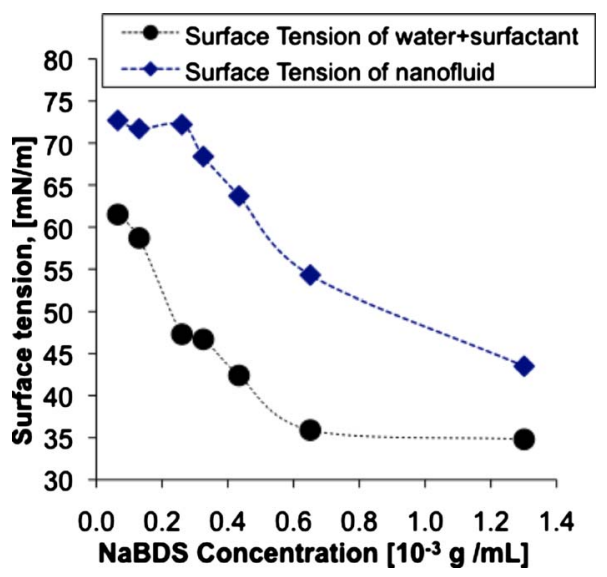

FIG. 4. (Color online) Surface tension of DI water+NaBDS and surface tension of $p$-SWNTs-nanofluid with NaBDS.

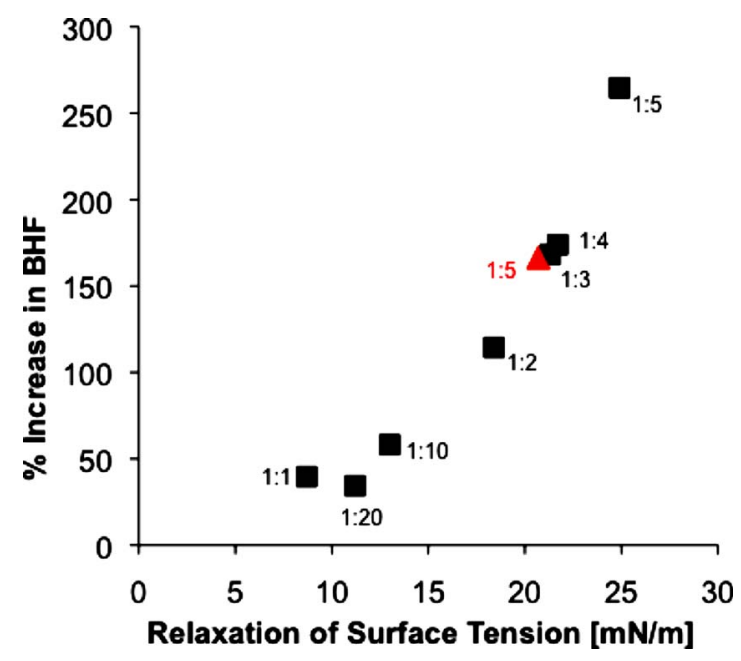

FIG. 5. (Color online) Percent increase in BHF for $p$-SWNTs (black square symbols) and $o$-SWNTs (red triangle symbol) as a function of relaxation of surface tension.

face tension and that of the surfactant solution increases at lower concentration, reaching a maximum for a concentration ratio of 1:5. As the surfactant concentration is increased further in the nanofluid, the surface tension difference starts decreasing again. At the highest concentration ratio of 1:1, the difference in surface tension is approximately $10 \mathrm{mN} / \mathrm{m}$, which is about the same as for a concentration ratio of 1:20. At any surfactant concentration, the difference between surface tension of nanofluid and pure fluid, termed as relaxation of surface tension, is solely due to the SWNTs in nanofluid. Thus, the relaxation of surface tension of nanofluid with respect to its base fluid is the driving force for the postponement of BHF of nanofluid $\left(\mathrm{BHF}_{N}\right)$ in pool boiling, as shown in Fig. 5. BHF reaches a maximum for a concentration of 1:5 (or $0.26 \mathrm{mg} / \mathrm{ml}$ ) NaDBS. This point represents the maximum adsorption of surfactant molecules on the nanotube walls. This plot also shows that the percent change in BHF steadily increases with the relaxation of surface tension, reaching a maximum of $265 \%$ enhancement for the case of $0.1 \%$ concentration that was maintained throughout all the experiments. Further work needs to be done to realize optimum CNT concentration and surfactant concentration.

${ }^{1}$ P. Keblinski, Y. Ding, and S. K. Das, Nanofluids: Fundamentals and Applications Conference, Copper Mountain, Colorado, 2007 (unpublished).

${ }^{2}$ H. Masuda, A. Ebata, K. Teramae, and N. Hishinuma, Netsu Bussei 4, 227 (1993).

${ }^{3}$ S. Lee, S. U. S. Choi, S. Li, and J. A. Eastman, ASME J. Heat Transfer 121, 280 (1999).

${ }^{4}$ H. Xie, J. Wang, T. Xi, Y. Liu, and F. Ai, J. Appl. Phys. 91, 4568 (2002).

${ }^{5}$ A. Gupta and R. Kumar, Appl. Phys. Lett. 91, 223102 (2007).

${ }^{6}$ N. N. Venkata Sastry, A. Bhunia, T. Sundararajan, and S. K. Das, Nanotechnology 19, 055704 (2008).

${ }^{7}$ P. Vassallo, R. Kumar, and S. D'Amico, Int. J. Heat Mass Transfer 47, 407 (2004).

${ }^{8}$ D. Milanova and R. Kumar, Appl. Phys. Lett. 87, 233107 (2005).

${ }^{9}$ D. Milanova and R. Kumar, ASME J. Heat Transfer 130, 042401 (2008).

${ }^{10}$ S. M. You, J. H. Kim, and K. H. Kim, Appl. Phys. Lett. 83, 3374 (2003).

${ }^{11}$ S. K. Das, N. Putra, and W. Roetzel, Int. J. Heat Mass Transfer 46, 851 (2003).

${ }^{12}$ H. Xie, H. Lee, W. Youn, and M. Choi, J. Appl. Phys. 94, 4967 (2003).

${ }^{13}$ H. Kim, J. Kim, and M. H. Kim, Int. J. Heat Mass Transfer 49, 5070 (2006).

${ }^{14}$ V. M. Wasekar and R. M. Manglik, ASME J. Heat Transfer 122, 708 (2000) 